\title{
Dengue Outbreak Prediction Based on Artificial Neural Networking Model Using Climatic Parameters
}

\section{Biplab Ghosh}

Indian Institute of Technology Guwahati

Monika Soni ( $\nabla$ monikasn12@gmail.com )

Regional Medical Research Centre Dibrugarh

\section{Research}

Keywords: artificial neural network, nonlinear models, Meteorological parameters, Dengue, Aedes mosquito

Posted Date: October 8th, 2020

DOI: https://doi.org/10.21203/rs.3.rs-86587/v1

License: (c) (i) This work is licensed under a Creative Commons Attribution 4.0 International License. Read Full License 
1 Title: Dengue outbreak prediction based on Artificial Neural networking model using

2 climatic parameters

3 Biplab Ghosh, $\mathrm{PhD}^{1}$ and Monika Soni, $\mathrm{PhD}^{2 *}$

4 Indian Institute of Technology, Guwahati, Assam-781039, India,

5 Email: biplabghosh22@gmail.com

$6 \quad{ }^{2}$ Assam Don Bosco University, Tepesia, Assam-781027, India,

7 Email: monikasn12@gmail.com

8 Corresponding author:

9 Dr. Monika Soni

10 Assistant Professor, Department of Life Science,

11 Department of Life Science,

12 Assam Don Bosco University, Tepesia, Assam-781027, India,

13 Email: monikasn12@gmail.com

14 Running title: Artificial Neural networking model for Dengue prediction

15 Word count: 2276

16 


\section{ABSTRACT}

18 Background: Dengue fever is a vector-borne tropical disease radically amplified by 30 times in

19 occurrence between 1960 and 2010. The upsurge is considered to be because of urbanization,

20 population growth and climate change. Therefore, Meteorological parameters (temperature,

21 precipitation and relative humidity) have impact on the occurrence and outbreaks of dengue

22 fever. There are not many studies that enumerate the relationship between the dengue cases in a

23 particular locality and the meteorological parameters. This study explores the relationship

24 between the dengue cases and the meteorological parameters. In prevalent localities, it is

25 essential to alleviate the outbreaks using modelling techniques for better disease control.

26 Methods: An artificial neural network (ANN) model was developed for predicting the number

27 of dengue cases by knowing the meteorological parameters. The model was trained with 7 years

28 of dengue fever data of Kamrup and Lakhimpur district of Assam, India. The practicality of the

29 model was corroborated using independent data set with satisfactory outcomes.

30 Findings: It was apparent from the sensitivity analysis that precipitation is more sensitive to the

31 number of dengue cases than other meteorological parameters.

32 Conclusion: This model would assist dengue fever alleviation and control in the long run.

33 Keywords: artificial neural network; nonlinear models; Meteorological parameters; Dengue;

$34 \quad$ Aedes mosquito 


\section{Background}

Dengue fever is a vector-borne disease that is one of the most important public health risks caused by the all four serotypes of dengue virus DENV-1, DENV-2, DENV-3 and DENV-4. Globally there are 100 to 400 million cases of infections annually in tropical and subtropical regions [1-4]. Aedes aegypti and Aedes albopictus mosquitoes are responsible for the diseases transmission $[1,3,5]$. As the Ae. aegypti mosquitoes have adjusted to urban settings, the control and mitigation of the disease has become very difficult [6-8]. The epidemics of dengue fever in India have turn out to be more common and have rapidly spread to new areas where dengue was not generally in existence [9]. Ensuing epidemics have been reported in different parts of India, especially in urban settings [10]. An important shift has been observed in the range of the dengue affected area where it is not constrained to urban areas only but has spread to rural expanses [11]. environmental aspects, unforeseen urbanization, population resistant issues and insufficient vector control actions which have shaped promising settings for dengue virus spread [9]. There are quite a few studies that conveyed the shifting spatial patterns in the transmission of dengue

51 fever with the causes, stretching from the increase mobility of individuals and goods, 52 proliferating vectors and pathogens to changes in climatic conditions [11-14]. Meteorological parameters like temperature, relative humidity and precipitation are important factors in mosquito population and disease transmission dynamics [15]. Temperature impacts the growth multiplicative performance of mosquitoes and precipitation delivers the water that helps as surroundings for larvae whereas humidity indulgence in prolonged existence of the mosquitoes and reduce the viral growth period leading to quicker virus replication and better transmission intensity [15-17]. It is also evident from the past literatures that the risk of dengue transmission is 
association between meteorological parameters and dengue fluctuates across the areas [20,21].

61 For tropical and subtropical region like India, dengue is highly seasonal but inadequate numbers

62 of researches have been carried out to estimate the effect of meteorological parameters on the

63 number of dengue cases.

There are some studies regarding linear and nonlinear methods simulating intricate

65 associations between climatic parameters and dengue fever incidence $[1,22,23]$. Though, the

66 linear methods are frequently inept to simulate complex relations between these parameters

67 [24,25]. Nonlinear methods have usually given away comparatively better results than linear 68 models [26-28].

The objective of the study is to establish a predictive model for the occurrence of dengue 70 fever and number of cases as a function of meteorological parameters like precipitation, 71 temperature and relative humidity. Two study areas were chosen in this study which were can be 72 studied independently. An artificial neural network (ANN) model was developed for predicting 73 the number of cases as a function of precipitation, temperature and relative humidity which was 74 additionally corroborated with the independent set of dengue cases. ANN use mixtures of 75 predictor variables of meteorological parameters to simulate association with target variable the 76 number of dengue fever cases. This model can be adjusted to integrate the data such that advance 77 the functional associations between meteorological parameters and the number of dengue fever 78 cases. The model would be useful for predicting the potential outbreaks of dengue fever with 79 known meteorological parameters and comprehend the dengue fever dynamics and advance 80 epidemiological observation which is the innovative feature of the study.

\section{Methodology}

\section{Description of the Study Area}


84 Brahmaputra, Assam, North-east India, with their geographic location between $25^{\circ} 31^{\prime} 3.434^{\prime \prime} \mathrm{N}$

85 and $26^{\circ} 44^{\prime} 55.122^{\prime \prime} \mathrm{N}$ Latitude and $90^{\circ} 56^{\prime} 14.324^{\prime \prime} \mathrm{E}$ and $92^{\circ} 6^{\prime} 10.578 " \mathrm{E}$ Longitude and $8626^{\circ} 31^{\prime} 3.254^{\prime \prime} \mathrm{N}$ and $27^{\circ} 28^{\prime} 32.471^{\prime \prime} \mathrm{N}$ Latitude and $93^{\circ} 51^{\prime} 54.414^{\prime \prime} \mathrm{E}$ and $94^{\circ} 57^{\prime} 24.874^{\prime \prime} \mathrm{E}$

87 Longitude respectively. Kamrup district has an average precipitation of $172 \mathrm{~cm}$ per year, with 88 annual average maximum temperatures of $32^{\circ} \mathrm{C}$ and minimum $19^{\circ} \mathrm{C}$, average relative humidity is $8982 \%$. Lakhimpur district has an average precipitation of $277 \mathrm{~cm}$ per year, with annual average 90 maximum temperatures of $29^{\circ} \mathrm{C}$ and minimum $18^{\circ} \mathrm{C}$ and the average relative humidity is $87 \%$.

91 For the months of May to October weather is wet and from December to March it is dry in both 92 the study areas.

\section{Dengue Fever Cases}

Dengue fever data for both the study areas were obtained from the department of 95 epidemiology from the year 2012-2018 for seven years. The phenology of dengue cases for both 96 the study areas is comparable, with cases typically increasing in August-October and decreasing 97 around December and January which follows the rainy season at both the study areas. The 98 number of dengue cases differs in different years due to various serotypes articulating 99 themselves in different times and the vulnerability of population with movement to affected 100 areas. The number of dengue cases in Lakhimpur district is minuscule in comparison to the 101 Kamrup district as one area has been chosen as exceedingly exposed to dengue fever and other is 102 slightly exposed.

\section{Meteorological data}


105 from the Indian Meteorological Department (IMD) from the year 2012-2018 for seven years.

106 Development of artificial neural network (ANN) model for Dengue Fever Cases

107 Neural networks comprise artificial neurons in a multi-layered architecture to institute 108 correlation between the input and the output parameters. A network is trained encompassing an 109 iterative process by which the network provides the suitable inputs along with an exact output for 110 each of the inputs [29]. In the wake of the training, the subsequent set has been learned with the 111 learning weights are slightly adjusted while each of the iterations carried out and the cycle is 112 settled when the appropriate weights have been achieved.

113 In the study, ANN was carried out to relate meteorological parameters with the number of 114 dengue cases. ANN has been used to predict the dengue cases for the study areas as a function of 115 precipitation, relative humidity and temperature. ANN toolbox available with MATLAB v $1162015 \mathrm{~A}$, has been used for the formation of the relationship between the number of dengue cases 117 and meteorological parameters precipitation, relative humidity and temperature. The network 118 was constructed and trained with various learning algorithms where multilayer feed-forward119 back-propagation network with Levenberg-Marquardt's learning rule found to be the most 120 competent and precise in estimating the output in comparison with the other algorithms with the 121 lowest mean square errors (MSE) with 5 neurons as shown in Fig. 1. The maximum possible 122 iterations had been set to 50,000 where the advancement in successive learning iterations was 123 determined by MSE, as expressed in Eq. 1,

$$
M S E=\frac{1}{N_{d}} \sum_{i=1}^{N}\left(O_{s}-O_{A}\right)^{2}
$$


125 where $\mathrm{O}_{\mathrm{S}}$ and $\mathrm{O}_{\mathrm{A}}$ were the simulated and predicted values respectively of the same unit and $\mathrm{N}_{\mathrm{d}}$ 126 was the total number of units. As obvious from figure 2, the number of neurons in the hidden

127 layer was optimized to have the least MSE. Therefore, the network structure was a 3-5-1

128 architecture with three neurons in the input layer, five neurons in the hidden layer and one 129 neuron in the output layer where non-linear tan-sigmoid transfer function was used for the nodal 130 connectors as shown in figure 2.

$131 \quad$ Results

132 Normalization of Input and Output

133 The normalization was conducted using the following expression given as,

$134 \quad S_{j}^{n}=2 \frac{S_{j}^{a}-S_{j}^{\min }}{S_{j}^{\max }-S_{j}^{\min }}-1$

135 where $S_{j}^{n}$ and $S_{j}^{a}$ were the $j^{\text {th }}$ values of input or output before and after normalization respectively

136 whereas $S_{j}^{\max }$ and $S_{j}^{\min }$ are the maximum and minimum values of all before normalization.

\section{Number of Hidden Neurons}

138 Investigation was conducted to find out the optimal number of neurons in dispensable in 139 the hidden layer. It was clear that the lowest MSE was in the case of Levenberg-Marquardt's 140 training function with 5neurons in the hidden layer. Therefore, a 3-5-1 ANN architecture had 141 been developed with three input neurons (precipitation, humidity and temperature) with one 142 output node (No of dengue cases) and five hidden neurons tabulated in table 1. 


\section{Overview and Performance of the ANN Architecture}

162 denormalized as,
The architecture of the ANN can adjust its performance in agreement with the precise problem. So, ANN has the capability to capture operative configuration from a particular dataset which is known as training where the connection weights of neurons change systematically to deliver the favored outcomes. The leading objective of the training is to discover the perfect connection weights which would generate minimum MSE. Capability of neural structure for training, Testing and Validation phase shown in figure 3.

\section{ANN Prediction Equation}

A model equation was outlined with the weights and biases attained from trained ANN. The mathematical equation linking the input variables and the output could be expressed as

$$
D_{n}=f_{\text {sig }}\left\{b_{0}+\sum_{k=1}^{h}\left[w_{k} \times f_{\text {sig }}\left(b_{h k}+\sum_{i=1}^{m} w_{i k} P_{i}\right)\right]\right\}(3)
$$

where $D_{n}$ was the normalized output variable, $f_{\text {sig }}$ is the sigmoid transfer function, $\mathrm{b}_{0}$ is the bias at the output layer; $W_{k}$ is the connection weight between $k^{\text {th }}$ node of hidden layer and the output node, $b_{h k}$ is the bias at the $k^{\text {th }}$ node of the hidden layer, $m$ is the number of input variables, $h$ is the number of neurons in the hidden layer, $W_{i k}$ is the connection weight between $i^{\text {th }}$ layer of input and $k^{\text {th }}$ node of hidden layer, $P_{i}$ is the normalized input variables.

With the values of the connection weights and biases tabulated in table 2-3 and subsequent ANN Prediction Equations of the model expressed in the table 4.

The $D_{n}$ value as acquired from table 5 was in between -1 and 1 and that required to be

$D=0.5\left(D_{n}+1\right)\left(D_{\max }-D_{\min }\right)+D_{\min }(4)$ 
164 where, $D_{\max }$ and $D_{\min }$ were the maximum and minimum value respectively of the dataset

\section{Sensitivity study of meteorology and dengue cases}

166

167

168

169

170

171

172

173

174

175

176

177

178 Input $_{X}=\sum_{Y=A}^{F} \frac{\mid \text { Hidden }_{X Y} \mid}{\sum_{Z=1}^{9} \mid \text { Hidden }_{Z Y} \mid}$ on their absolute values.

The goal of a sensitivity analysis is comparable to evaluating relative importance of explanatory variables, with a few differences. The relationships between explanatory and response variables as described by the model in the hope that the neural network has explained some real-world phenomenon.It is noteworthy for the choice of inducing input variables so as to give their ranking according to their importance. Garson's algorithm was used in this study to find the importance of inputs [28]. Using Garson's algorithm, we can get an idea of the magnitude and sign of the relationship between variables relative to each other. At first the inputhidden and hidden-output weights were disjointed, and the absolute values of the weights were used to differentiate the rank of input parameters. The three meteorological parameters are the inputs for the study. In the method, the products of the input-hidden and hidden-output connection weights were measured. The outcome is the standing of the input variables centered

Accordingly, the above expression represents the estimation of variable importance for 180 predictor variable $\mathrm{X}$ (where $\mathrm{X}=1-3$ ), using the weights connecting each of the input neurons $\mathrm{Z}$ 181 (where $\mathrm{Z}=1-3$ ) to each of the hidden neurons $\mathrm{N}$ (where $\mathrm{N}=1-5$ ), and the latter to the single 182 output neuron. The result of the sensitivity analysis conducted with the Garson's method to 183 deliver the importance ranking to the input parameters was shown in table 5. Relative humidity 
184 has been found to be the most important input parameter followed by temperature and 185 precipitation.

\section{Discussion}

There are fairly a few studies that conveyed the association between meteorological

188 parameters and dengue fever though it vacillates across the areas [18-21]. As the meteorological

189 parameters like temperature, relative humidity and precipitation significantly influences

190 mosquito population and spread of the disease, establishing a predictive model for the occurrence

191 of dengue fever is essential [15]. Though there are studies simulating associations between

192 climatic parameters and dengue fever incidence with both linear and nonlinear methods,

193 nonlinear methods like ANN have typically provided with relatively better results [22,26-28].

194 ANN model has also been used in Thailand, Singapore, Malaysia, North America to predict 195 dengue fever cases with high accuracies [25,30-32]. The present study is the first such approach

196 in India in that context with an ANN model has been developed for predicting the number of 197 cases as a function of precipitation, temperature and relative humidity which was additionally 198 corroborated with the independent set of dengue cases with two study areas chosen which were 199 studied independently. A model equation has been developed with ANN for determining the 200 number of dengue cases as a function of three meteorological parameters which would be useful 201 for predicting the potential outbreaks of dengue fever and advance epidemiological observation. 202 The sensitivity analysis of the meteorological parameters for predicting the vulnerability of 203 dengue fever is also first such effort in the context of India.

\section{Conclusions}

The number of dengue cases was modeled in this study using meteorological parameters with a nonlinear neural network method. An Artificial neural network has been developed with a 
207 feed forward back propagation neural network to predict the number of dengue cases towards 208 three meteorological parameters. The ANN model with the five hidden neurons is the optimal 209 model based on training and testing data set. A model equation has been developed based on the 210 trained weights of the ANN for determining the number of dengue cases as a function of three 211 meteorological parameters. Based on sensitivity analysis as per the Garson's algorithm 212 approaches, relative humidity is the most important input parameters for predicting the 213 vulnerability of dengue fever.

214 There were numerous influences that were not deliberated in the predictive ability of the 215 ANN models. Additional studies are desirable to include population vulnerability to dengue, 216 vector and dengue virus dynamics into the models which advance the ability of simulations and 217 comprehend related diseases that be influenced by meteorological changes. This is also 218 perceived that meteorological parameters are not the only issues influencing the sudden changes 219 in the dengue cases affecting the precision of the model. Future studies should increase the time 220 period to comprehend the seasonal and spatial variances across dengue fever prevalent regions 221 and can apply ANNs in that region to predict the number of cases.

222 Author's contribution: BG has worked on the modeling software and designed the prediction 223 model. MS helped in framing the manuscript, worked on dengue disease transmission pattern 224 and acquired data from National Vector Borne Disease Control Programme (NVBDCP), 225 Guwahati and regional metrological centre. Both the authors have read and approved the 226 manuscript.

\section{Declarations:}


228 Ethics approval and consent to participate: Ethical approval and consent to participate is not 229 necessary for the publication.

230 Consent for publication: Authors declare consent for publication for this manuscript.

231 Competing interests: Authors declare there is no any conflict of interest for submitting and 232 publishing of this article

233 Funding: There was no source of funding to carry out this work

234 Acknowledgement: Authors acknowledge to NVBDCP, Guwahati, India and Dr. J. Sarma, 235 District Epidemiologist, IDSP, Lakhimpur, India for providing archived data of dengue cases for 236 the past 10 years. Authors would also like to thanks to Assam Don Bosco University for 237 providing necessary support in data acquisition from different sources. We would like to thanks 238 Regional Metrological Centre, LGBI Airport, Guwahati to provide necessary metrological data.

239 References

240 1. Bhatt S, Gething PW, Brady OJ, Messina JP, Farlow AW, Moyes CL, et al. The global distribution and burden of dengue. Nature. 2013; 496:504-507.

2. Halstead SB. Dengue. The lancet.2007; 370:1644-1652.

3. Murray NEA, Quam MB, Wilder-Smith A. Epidemiology of dengue: past, present and future prospects. Clinical epidemiology. 2013; 5:299.

4. Mustafa M, Rasotgi V, Jain S, Gupta V. Discovery of fifth serotype of dengue virus (DENV-5): A new public health dilemma in dengue control. Medical Journal Armed Forces India.2015; 71:67-70. 
5. Shepard DS, Coudeville L, Halasa YA, Zambrano B, Dayan GH. Economic impact of dengue illness in the Americas. The American journal of tropical medicine and hygiene. 2011; 84:200-207.

6. Cheong W. Preferred Aedes aegypti larval habitats in urban areas. Bulletin of the World Health Organization.1967; 36: 586.

7. GRATZ NG. Emergency Control Of Aedes Aegyp as A Disease Vector In Urban Areas. J Ame Mosq Control Assoc.1991;7:353-365.

8. Gubler DJ. Dengue and dengue hemorrhagic fever. Clinical microbiology reviews.1998;11:480-496.

9. Chakravarti A, Arora R, Luxemburger C. Fifty years of dengue in India. Transactions of the Royal Society of Tropical Medicine and Hygiene. 2012; 106: 273-282.

10. Chaturvedi U, Nagar R. Dengue and dengue haemorrhagic fever: Indian perspective. Journal of biosciences.2008; 33:429-441.

11. Arunachalam N, Murty U, Kabilan L, Balasubramanian A, Thenmozhi V, Narahari D, Ravi A, Satyanarayana K. Studies on dengue in rural areas of Kurnool District, Andhra Pradesh, India. Journal of the American Mosquito Control Association. 2004; 20:8790.

12. Fischer D, Thomas SM, Beierkuhnlein C. Temperature-derived potential for the establishment of phlebotomine sandflies and visceral leishmaniasis in Germany. Geospatial Health.2010; 5:56-69.

13. Pfeffer M, Dobler G. What comes after bluetongue--Europe as target for exotic arboviruses. Berliner und Munchener tierarztliche Wochenschrift. 2009;122:458-466. 
14. Randolph SE, Rogers DJ. The arrival, establishment and spread of exotic diseases: patterns and predictions. Nature Reviews Microbiology. 2010;8:361-371

15. Patz JA, Martens W, Focks DA, Jetten TH. Dengue fever epidemic potential as projected by general circulation models of global climate change. Environmental health perspectives. 1998;106:147-153.

16. Hopp MJ, Foley JA. Global-scale relationships between climate and the dengue fever vector, Aedes aegypti. Climatic change.2001;48: 441-463.

17. Tseng WC, Chen CC, Chang CC, Chu YH. Estimating the economic impacts of climate change on infectious diseases: a case study on dengue fever in Taiwan. Climatic Change. 2009;92:123-140.

18. Gonçalves VN, Rebelo J. Epidemiological characteristics of dengue in the Municipality of Sao Luis, Maranhao, Brazil, 1997-2002. Cadernos de Saúde Pública.2004;20:14241431.

19. Indaratna K, Hutubessy R, Chupraphawan S, Sukapurana C, Tao J, Chunsutthiwat S, et al. Application of geographical information systems to co-analysis of disease and economic resources: dengue and malaria in Thailand. The Southeast Asian journal of tropical medicine and public health. 1998;29:669-684.

20. Arcari P, Tapper N, Pfueller S. Regional variability in relationships between climate and dengue/DHF in Indonesia. Singapore Journal of Tropical Geography.2007; 28: 251-272.

21. Thammapalo S, Chongsuvivatwong V, Geater A, Dueravee M. Environmental factors and incidence of dengue fever and dengue haemorrhagic fever in an urban area, Southern Thailand. Epidemiology \& Infection.2008; 136:135-143. 
22. de Castro Medeiros LC, Castilho CAR, Braga C, de Souza WV, Regis L, Monteiro AMV. Modeling the dynamic transmission of dengue fever: investigating disease persistence PLOS neglected tropical diseases.2011; 5.

23. Racloz V, Ramsey R, Tong S, Hu W. Surveillance of dengue fever virus: a review of epidemiological models and early warning systems. PLoS neglected tropical diseases.2012; 6 .

24. Laureano-Rosario AE, Garcia-Rejon JE, Gomez-Carro S, Farfan-Ale JA, MullerKarger FE. Modelling dengue fever risk in the State of Yucatan Mexico using regionalscale satellite-derived sea surface temperature. Acta tropica. 2017; 172:50-57. Carro S, Farfan-Ale J, et al. Application of artificial neural networks for dengue fever outbreak predictions in the northwest coast of Yucatan Mexico and San Juan, Puerto Rico. Tropical medicine and infectious disease.2018; 3:5.

26. Husin NA, Salim N. Modeling of dengue outbreak prediction in Malaysia: a comparison of neural network and nonlinear regression model. 2008 International Symposium on Information Technology IEEE.2008;1-4.

27. Parham PE, Michael E. Modeling the effects of weather and climate change on malaria transmission. Environmental health perspectives.2010; 118:620-626.

28. Wu Y, Lee G, Fu X, Hung T. Detect climatic factors contributing to dengue outbreak based on wavelet, support vector machines and genetic algorithm.2008.

29. Garson DG. Interpreting neural network connection weights.1991.

30. Aburas HM, Cetiner B.G, Sari M. Dengue confirmed-cases prediction: A neural network model. Expert Systems with Applications.2010; 37:4256-4260. 
31. Hwang S, Clarite DS, Elijorde FI, Gerardo BD, Byun Y. A web-based analysis for dengue tracking and prediction using artificial neural network. SERSC: ASTL. 2016;122:160-164.

32. Rachata N, Charoenkwan P, Yooyativong T, Chamnongthal K, Lursinsap C, Higuchi K. Automatic prediction system of dengue haemorrhagic-fever outbreak risk by using entropy and artificial neural network, 2008 International Symposium on Communications and Information Technologies IEEE. 2008; 210-214. 


\section{Figures Legends:}

326 Figure1.Training program of hidden layer transfer functions with MSE data

327 Figure2. Structure of the Neural Network

328 Figure3. Capability of neural structure for training, Testing and Validation phase 
Figures

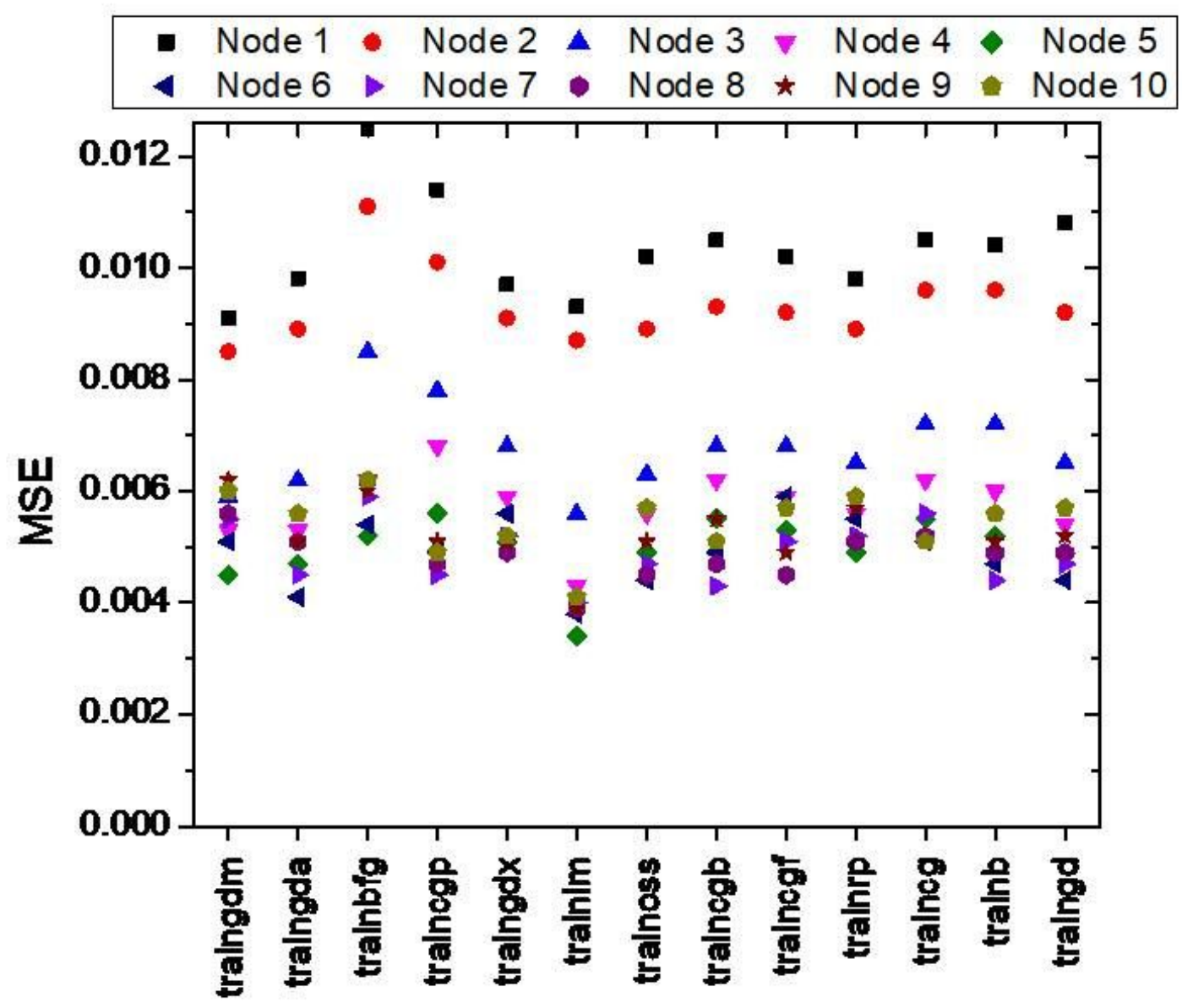

Figure 1.Training program of hidden layer transfer functions with MSE data

Figure 1

Figure 1 
Fidden Layers

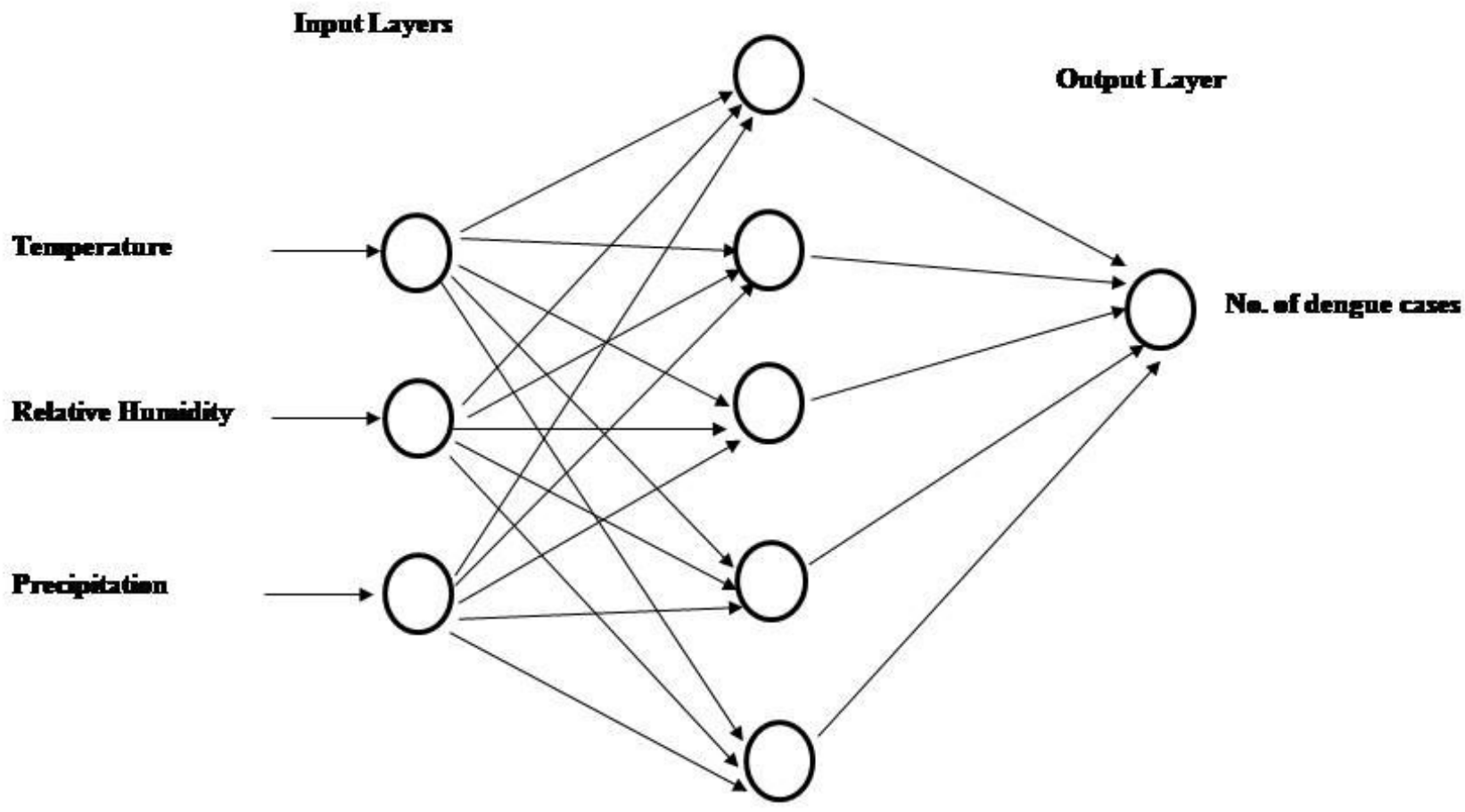

Figure 2.Structure of the Neural Network

Figure 2

Figure 2 

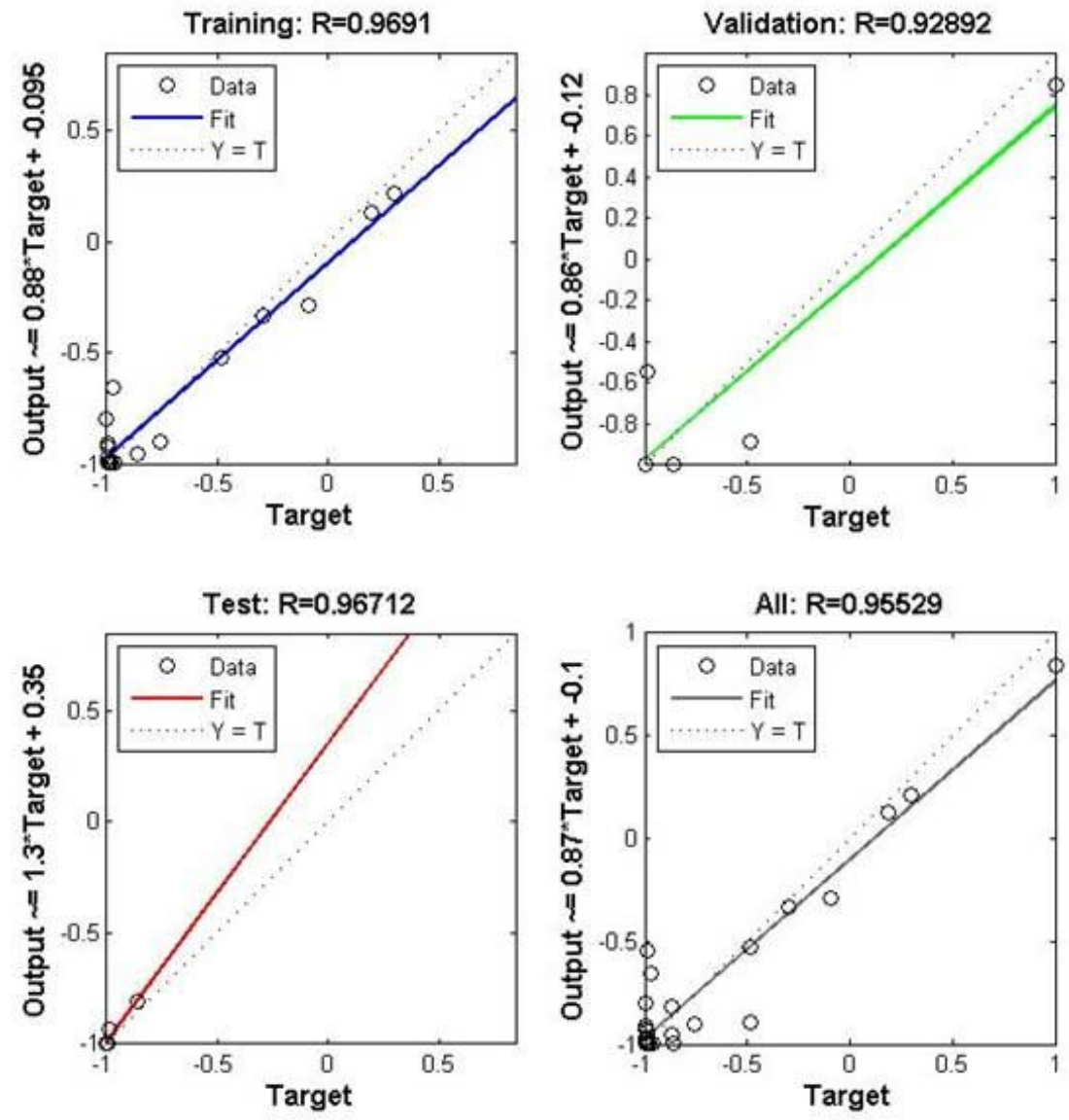

Figure 3. Capability of neural structure for training, Testing and Validation phase

Figure 3

Figure 3

\section{Supplementary Files}

This is a list of supplementary files associated with this preprint. Click to download.

- Table1.docx

- Table2.docx

- Table3.docx

- Table4.docx

- Table5.docx 